\title{
A Case of Multifaceted Assessment in an Elderly Patient With Acute Decompensated Heart Failure
}

\author{
Hideto Sako ${ }^{\mathrm{a}}$, Midori Miyazaki ${ }^{\mathrm{a}}$, Yasunori Suematsu ${ }^{\mathrm{a}}$, Rie Koyoshi ${ }^{\mathrm{b}}$, Yuhei Shiga ${ }^{\mathrm{a}}$, \\ Takashi Kuwano ${ }^{\text {a }}$, Ken Kitajima ${ }^{\text {a }}$, Atsushi Iwata ${ }^{a}$, Katsura Yorinaga ${ }^{c}$, \\ Kanta Fujimi ${ }^{\mathrm{a}, \mathrm{d}}$, Shin-ichiro Miura ${ }^{\mathrm{a}, \mathrm{e}, \mathrm{f}}$
}

\begin{abstract}
Heart failure (HF) is a common health problem worldwide, including in Japan. Unfortunately, patient outcomes remain poor, with a 5 -year survival rate of approximately $50 \%$. Therefore, we need to assess the precise conditions, including cardiac function, in patients with HF, particularly in the elderly. We performed a multifaceted assessment in an elderly patient with HF on admission and at discharge using eight different evaluations (the mean life expectancy using the Seattle Heart Failure Model (SHFM), the severity of dementia, nutrition, medication adherence, biomarker (the level of brain natriuretic peptide in blood), sociality, performance and comorbidity). Each parameter was scored on a 5 -point scale (excellent $=5$ points; good $=4$ points; fair (average) $=3$ points; poor $=2$ points; failure $=1$ point; maximum total points of 40 ) (Fukuoka University Heart Failure Scoring System, FUFS). An 86-year-old male patient who complained of dyspnea and lower-leg edema was admitted to our university hospital due to acute decompensated HF. After treatment, his symptoms improved, as did his cardiothoracic ratio, plural effusion and pulmonary congestion, and he exhibited compensated HF. His total score improved from 28 to 32 points, and his mean life expectancy using SHFM increased from 4.9 to 5.4 years. We evaluated the precise conditions using a multifaceted assessment strategy in an elderly patient with HF. The strategy was useful for evaluate the patient's condition in this case.
\end{abstract}

Keywords: Heart failure; Dementia; Nutrition assessment; Medication adherence; Biomarker; Comorbidity

Manuscript submitted November 22, 2017, accepted December 12, 2017

aDepartment of Cardiology, Fukuoka University School of Medicine, Fukuoka, Japan

bDivision of Medical Safety Management, Fukuoka University Hospital, Fukuoka, Japan

'Division of Nursing, Fukuoka University Hospital, Fukuoka, Japan ${ }^{\mathrm{d} D i v i s i o n}$ of Rehabilitation, Fukuoka University Hospital, Fukuoka, Japan eDepartment of Molecular Cardiovascular Therapeutics, Fukuoka University School of Medicine, Fukuoka, Japan

${ }^{f}$ Corresponding Author: Shin-ichiro Miura, Department of Cardiology, Fukuoka University School of Medicine, 7-45-1 Nanakuma, Jonan-Ku, Fukuoka 814-0180, Japan.Email: miuras@cis.fukuoka-u.ac.jp

doi: https://doi.org/10.14740/cr640w

\section{Introduction}

Heart failure (HF) is a major health problem worldwide, including in Japan. Unfortunately, patient outcomes remain poor, with a 5-year survival rate of approximately $50 \%$ [1]. In Japan, the number of outpatients with HF is expected to increase rapidly, and there will be an estimated 1.2 million HF patients in 2025 [2]. In addition, the number of Japanese HF patients aged $65-84$ years will gradually increase until a peak in 2025. The elderly face various challenges besides cardiac dysfunction, such as impaired cognitive function, reduced capacity for physical exercise, family relations, the need for nursing care, etc. Although it is important for elderly patients to receive appropriate medications to treat HF, we need to assess the precise conditions in these patients, including cardiac function. In this report, we describe the application of a multifaceted assessment using eight different evaluations (the mean life expectancy in HF using the Seattle Heart Failure Model (SHFM) [3], the severity of dementia [4, 5], nutrition [6], medication adherence [7], biomarker [8], sociality [9], performance $[10,11]$ and comorbidity $[12,13])$ in an elderly patient with HF.

\section{Case Report}

An 86-year-old male complained of dyspnea and lower-leg edema. He had had hypertension for decades and had undergone a stent graft procedure for abdominal aortic aneurysm. He had dyspnea at night and lower-leg edema for the preceding 4 days. Since these symptoms were sustained, he was admitted to our university hospital due to suspected acute decompensated HF. His level of consciousness was good. His height and body weight were $162 \mathrm{~cm}$ and $46.2 \mathrm{~kg}$, respectively. Although his blood pressure was $154 / 72 \mathrm{~mm} \mathrm{Hg}$, other vital signs were normal (heart rate: 95 beats $/ \mathrm{min}$, regular; respiratory rate: $19 / \mathrm{min}$ ). Heart sounds showed systolic ejection murmur on the left border of the second intercostal sternum. Coarse crackles were noted in the bilateral lung fields. A 12-lead electrocardiogram showed normal sinus rhythm (77 beats/min), flat $\mathrm{T}$ wave and a QRS duration of 90 ms. With regard to biochemical parameters in blood, creatinine, troponin-I and coagulative functions were all normal, 


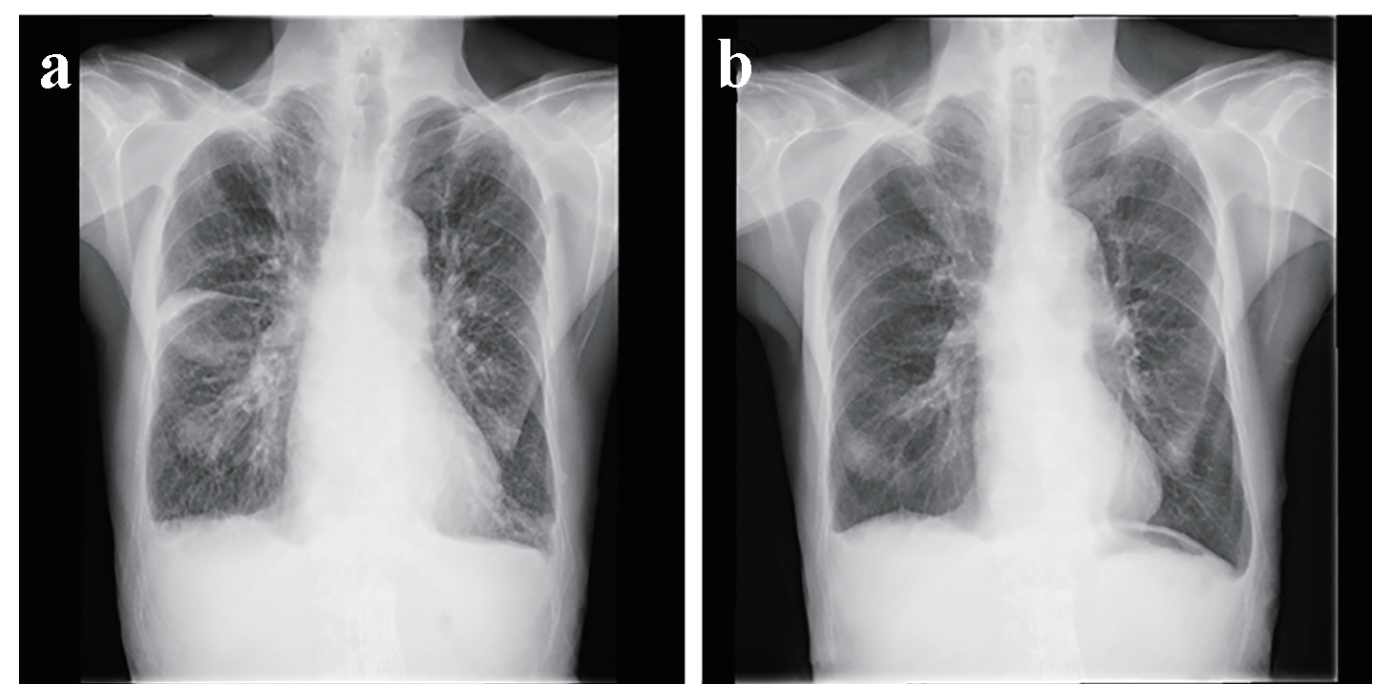

Figure 1. Chest radiography on admission (a) and at discharge (b).

Table 1. A Multifaceted Assessment Using Eight Different Evaluations (A-H)

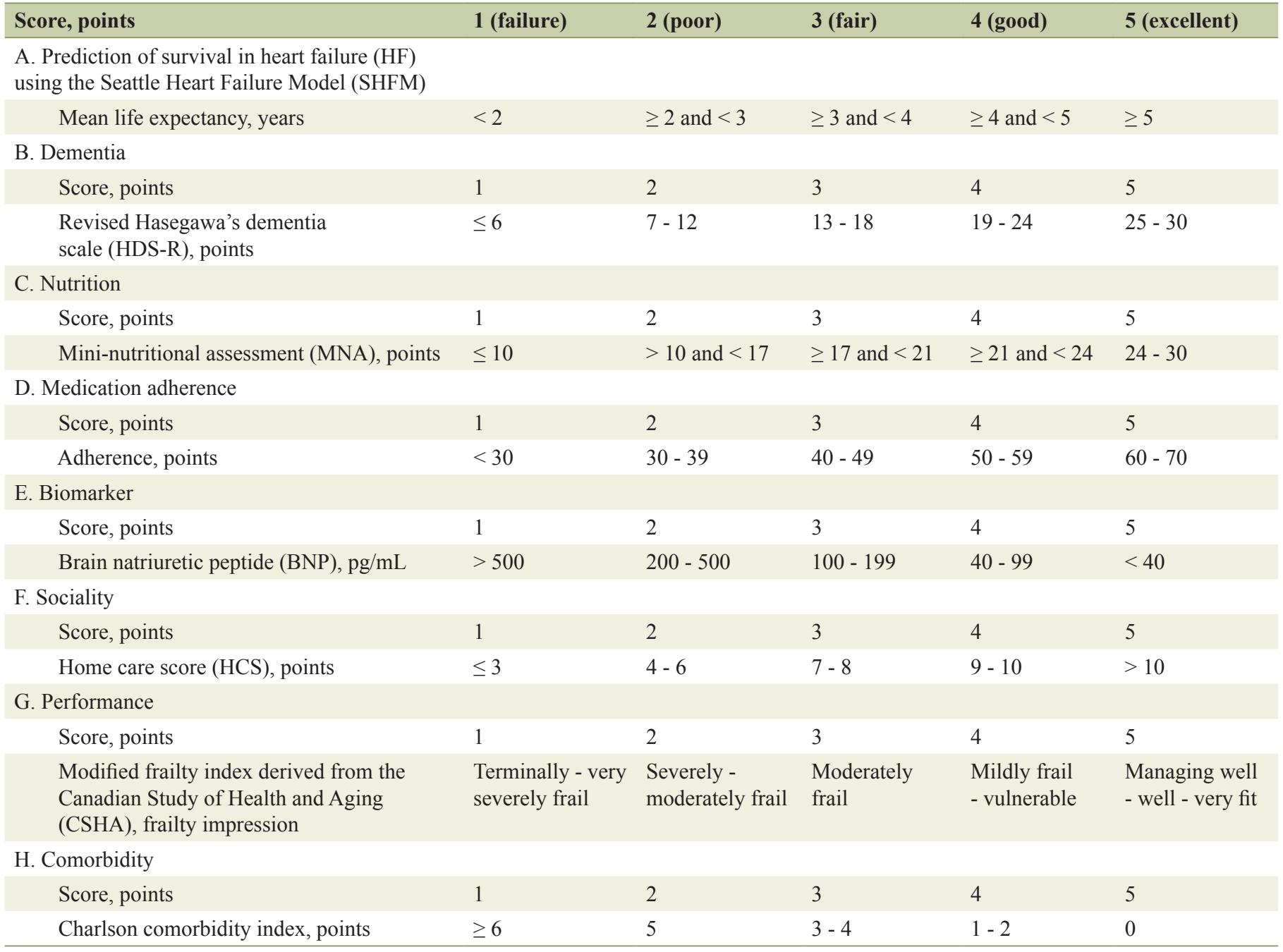



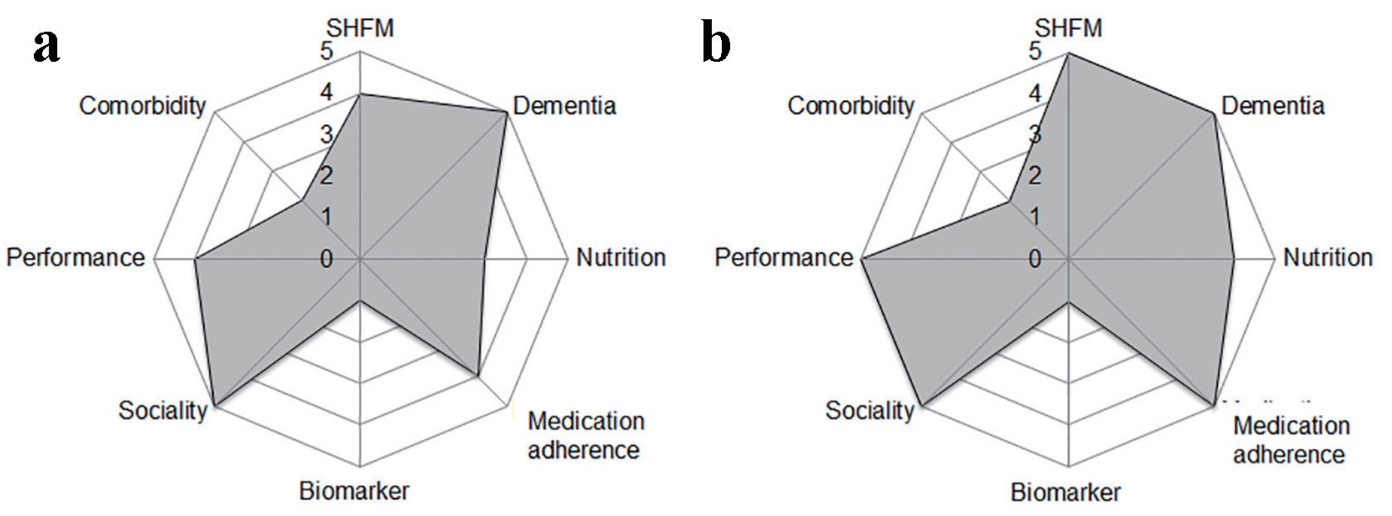

Figure 2. A multifaceted assessment on admission (a) and at discharge (b).

whereas the levels of white blood cell count $(10,500 / \mathrm{mL})$ and C-reactive protein $(1.62 \mathrm{mg} / \mathrm{dL})$ were elevated, along with the brain natriuretic peptide (BNP) $(853 \mathrm{pg} / \mathrm{mL})$. The levels of albumin $(3.9 \mathrm{~g} / \mathrm{dL})$, creatine kinase $(59 \mathrm{U} / \mathrm{L})$ and hemoglobin $(11.7 \mathrm{~g} / \mathrm{dl})$ in blood were decreased. Chest radiography showed cardiac dilatation (cardiothoracic ratio of $58 \%$ ) (Fig. 1a), and plural effusion and pulmonary congestion were observed. Echocardiography showed a left ventricular ejection fraction of $58 \%$, and mild aortic valve stenosis and regurgitation. The patient started to receive furosemide $40 \mathrm{mg} /$ day, benidipine $2 \mathrm{mg}$ /day and candesartan $4 \mathrm{mg}$ /day, and HF gradually became compensated. On day 14 , the cardiothoracic ratio decreased to $45 \%$ and plural effusion and pulmonary congestion were improved (Fig. 1b). Although he was classified as New York Heart Association (NYHA) IV on admission, he was NYHA II at discharge on day 15.

We performed a multifaceted assessment using eight different evaluations at both admission and discharge (Table 1). The evaluations included the mean life expectancy in HF using the SHFM [3], the severity of dementia [4, 5], nutrition [6], medication adherence [7], biomarker (the level of BNP in blood) [8], sociality [9], performance [10, 11] and comorbidity $[12,13]$. Each parameter was scored on a 5-point scale (excellent $=5$ points; good $=4$ points; fair (average) $=3$ points; poor $=2$ points; failure $=1$ point; maximum total points of 40) (Fukuoka University Heart Failure Scoring System, FUFS). The mean life expectancy according to SHFM [3] on admission and at discharge was 4.9 years ( 4 points) and 5.4 years ( 5 points), respectively (Table 1A) (Fig. 2). The severity of dementia was assessed using revised Hasegawa's dementia scale (HDS-R) $[4,5]$, and the scores on admission and at discharge were 5 and 5 points, respectively (Table 1B) (Fig. 2). Nutrition was assessed using mini-nutritional assessment (MNA) with some modification of scoring [6]; the value on admission (3 points) improved to 4 points at discharge (Table 1C) (Fig. 2). The scores for medication adherence (Table 1D and Table 2) [7] on admission and at discharge were 4 and 5 points, respectively (Fig. 2). Although BNP levels (Table 1E) [8] decreased to $523 \mathrm{pg} / \mathrm{mL}$ at discharge, there was no change in the number of points assigned to the biomarker evaluation. There was no problem in sociality as assessed by the home care score (HCS) with some modification of scoring [9]; the scores at both ad- mission and discharge were 5 (Table 1F) (Fig. 2). Performance as assessed by a modified frailty index derived from the Canadian Study of Health and Aging (modified FI-CSHA) [10, 11] improved from 4 points on admission to 5 points at discharge (Table 1G) (Fig. 2). There were no changes in the number of points assigned to comorbidity using the Charlson comorbidity index between admission and discharge (Table 1H) (Fig. 2) $[12,13]$ because the patient had both pulmonary emphysema and prostate cancer. Overall, the total score improved from 28 to 32 points.

\section{Discussion}

We evaluated the precise conditions, including cardiac function, in an elderly patient with acute decompensated HF using a multifaceted assessment with eight different evaluations. Elderly patients more frequently experience comorbidities, frailty, polypharmacy and cognitive impairment, all of which can have a significant impact on clinical outcomes. Thus, patients require proper evaluation to best assess and plan their care needs.

In this patient, the total points using a multifaceted assessment improved from 28 to 32 . Interestingly, the mean life expectancy (SHFM) improved with short-term care during hospitalization. Although nutrition (MNA), medication adherence and performance (modified FI-CSHA) each improved by only 1 point, these three factors are significantly associated with the prognosis in patients with HF according to previous reports [14-17]. A poor nutritional status as assessed using the controlling nutritional status score was significantly associated with inflammation and predicts poor outcomes in patients with HF [14]. Medication adherence and social support, both independently and in combination, predicted cardiac event-free survival in patients with HF [15]. A high prevalence of frailty has been reported in older patients with HF [16]. Increased frailty has been associated with an increased risk of falls, hospitalization, nursing home admission and mortality, with poorer outcomes [17]. In addition, although dementia and delirium are associated with worse clinical outcomes, including a longer duration of hospitalization and increased inpatient and 1-year 
Table 2. Medication Adherence Scale

Points

A. Coordination with medical staff regarding medication

1) Regarding medicine, I can ask medical staff such as medical doctors without hesitation. 1 - 5

2) Regarding medicine, I can share my previous treatment course with medical staff such as medical doctors. 1 - 5

3) Regarding medicine, I can share my thoughts and goals with medical staff such as medical doctors. 1 - 5

B. Aggressiveness in acquiring and using information about medication

4) I ask if there is something I do not know about my medication.

5) I report side effects of medicines, allergic symptoms, and unusual symptoms. 1 - 5

6) I know about my medicines and why they are needed. $1-5$

7) I use approaches to continue taking my medicine (ingenuity in daily life, etc.) 1 - 5

8) I am looking for and using information necessary for my medicines. 1 - 5

C. Conviction regarding medication and degree of cooperation

9) I am convinced about the necessity of my medicines. $1-5$

10) The use of medicines is part of my life, like eating meals and brushing my teeth. $1-5$

11) My family members and other people offer no resistance to helping me, such as by making phone calls regarding 1 - 5 my medications.

D. Compliance with medication

12) Medication has been used for the indicated number of times or tablets over the last three weeks. 1 - 5

13) Medication has been used for the indicated time over the last three weeks. $1-5$

14) I did not stop taking my medicines at my own discretion. $1-5$

Total points $14-70$

1 point: not applicable; 2 point: not very true; 3 point: neither agree nor disagree; 4 point: slightly true; 5 point: applies.

mortality [18], and patients with HF who receive home-based rehabilitation services make significant improvements in activities of daily living [19], this patient did not have dementia or delirium and had a high HCS.

We also assessed comorbidities on admission and at discharge. Although we appropriately treated HF during hospitalization, there were no changes in the points assigned to comorbidity. Up to $75 \%$ of patients aged $>65$ years will have multiple chronic conditions that will impact HF management [20], and comorbidity is one of the strongest independent predictors of re-hospitalization and mortality [21]. Thus, we must consider caring for comorbidities during hospitalization. In addition, the mean life expectancy improved at discharge. The SHFM provides an accurate estimate of 1-, 2-, and 3-year survival with the use of easily obtained clinical, pharmacological, device, and laboratory characteristics [3]. Since SHFM does not include assessments of dementia, nutrition, medication adherence, sociality or performance, we also need to carefully evaluate these factors in addition to any therapies (both pharmacological and with the use of devices) and laboratory parameters.

The prevalence of HF with preserved ejection fraction (HFpEF) is increasing [22]. In the present case, LVEF was $58 \%$, and we gave a diagnosis of $\mathrm{HFpEF}$, probably due to HTN. Since HFpEF is a condition for which no treatment has been proven to alter clinical outcomes, it is important to evaluate the patient's condition. When patients with HFpEF were further categorized as having relatively low LVEF (HFrlEF;
EF $40-60 \%$ ) or relatively high LVEF (HFrhEF; EF $\geq 60 \%$ ), the BNP level was shown to have prognostic value in HFrlEF, but not in HFrhEF [23]. The BNP level may also be important for predicting the prognosis in HF patients. Since there was no change in the number of points assigned to BNP and its level was still high at discharge, more aggressive treatment might be needed, even though this patient is very elderly.

Comprehensive cardiac rehabilitation (CR) has been shown to improve quality of life in addition to cardiac function in patients with HF [24]. This patient immediately started CR. CR may also improve the patient's condition using eight evaluations, even though $\mathrm{CR}$ was only performed about 2 weeks.

To the future, it is necessary to evaluate the usefulness of a multifaceted assessment strategy in clinical trial, and it may subsequently give appropriate therapies.

\section{Conclusion}

We evaluated the precise conditions using a multifaceted assessment strategy in an elderly patient with HF. The strategy was useful for evaluating the patient's condition in this case.

\section{Financial Support}

None. 


\section{Conflict of Interest}

SM is a Director of NPO Clinical and Applied Science, Fukuo$\mathrm{ka}$, Japan, and belongs to the Department of Molecular Cardiovascular Therapeutics supported by MSD, Co. LTD.

\section{References}

1. Mozaffarian D, Benjamin EJ, Go AS, Arnett DK, Blaha MJ, Cushman M, de Ferranti S, et al. Heart disease and stroke statistics - 2015 update: a report from the American Heart Association. Circulation. 2015;131(4):e29-322.

2. Okura Y, Ramadan MM, Ohno Y, Mitsuma W, Tanaka K, Ito M, Suzuki K, et al. Impending epidemic: future projection of heart failure in Japan to the year 2055. Circ J. 2008;72(3):489-491.

3. Levy WC, Mozaffarian D, Linker DT, Sutradhar SC, Anker SD, Cropp AB, Anand I, et al. The Seattle Heart Failure Model: prediction of survival in heart failure. Circulation. 2006;113(11):1424-1433.

4. Kato S, Simogaki H, Onodera A, Ueda H, Oikawa K, Ikeda K, et al. Preparation of Revised Hasegawa's Dementia Scale (HDS-R). Jpn J Geriatr Psychiatr. 1991;2:13391347. in Japanese.

5. Imai Y, Hasegawa K. The revised Hasegawa's Dementia Scale (HDS-R) - evaluation of its usefulness as a screening test for dementia. J Hong Kong Coll Psychiatr. 1994;4(SP2):20-24.

6. Vellas B, Villars H, Abellan G, Soto ME, Rolland Y, Guigoz Y, Morley JE, et al. Overview of the MNA - Its history and challenges. J Nutr Health Aging. 2006;10(6):456463; discussion 463-455.

7. Ueno H, Kamazaki Y, Ishikawa H. Reliability and validity of medication adherence scale for patients with chronic disease in Japan. JJHEP. 2014:22:13-29. in Japanese.

8. Fukuda H, Suwa H, Nakano A, Sakamoto M, Imazu M, Hasegawa T, Takahama H, et al. Non-linear equation using plasma brain natriuretic peptide levels to predict cardiovascular outcomes in patients with heart failure. Sci Rep. 2016;6:37073.

9. Matsugi A, Tani K, Tamaru Y, Yoshioka N, Yamashita A, Mori N, Oku K, et al. Prediction of Advisability of Returning Home Using the Home Care Score. Rehabil Res Pract. 2015;2015:501042.

10. Arya S, Kim SI, Duwayri Y, Brewster LP, Veeraswamy R, Salam A, Dodson TF. Frailty increases the risk of 30-day mortality, morbidity, and failure to rescue after elective abdominal aortic aneurysm repair independent of age and comorbidities. J Vasc Surg. 2015;61(2):324-331.

11. Mitnitski AB, Mogilner AJ, Rockwood K. Accumulation of deficits as a proxy measure of aging. ScientificWorldJournal. 2001;1:323-336.

12. Charlson ME, Pompei P, Ales KL, MacKenzie CR. A new method of classifying prognostic comorbidity in longitudinal studies: development and validation. J Chronic Dis.
1987;40(5):373-383.

13. Quan H, Li B, Couris CM, Fushimi K, Graham P, Hider $\mathrm{P}$, Januel JM, et al. Updating and validating the Charlson comorbidity index and score for risk adjustment in hospital discharge abstracts using data from 6 countries. Am J Epidemiol. 2011;173(6):676-682.

14. Nakagomi A, Kohashi K, Morisawa T, Kosugi M, Endoh I, Kusama Y, Atarashi H, et al. Nutritional Status is Associated with Inflammation and Predicts a Poor Outcome in Patients with Chronic Heart Failure. J Atheroscler Thromb. 2016;23(6):713-727.

15. Wu JR, Frazier SK, Rayens MK, Lennie TA, Chung ML, Moser DK. Medication adherence, social support, and event-free survival in patients with heart failure. Health Psychol. 2013;32(6):637-646.

16. Clegg A, Young J, Iliffe S, Rikkert MO, Rockwood K. Frailty in elderly people. Lancet. 2013;381(9868):752762.

17. Song X, Mitnitski A, Rockwood K. Prevalence and 10year outcomes of frailty in older adults in relation to deficit accumulation. J Am Geriatr Soc. 2010;58(4):681-687.

18. Alosco ML, Spitznagel MB, Cohen R, Sweet LH, Colbert LH, Josephson R, Hughes J, et al. Reduced cognitive function predicts functional decline in patients with heart failure over 12 months. Eur J Cardiovasc Nurs. 2014;13(4):304-310.

19. Asiri FY, Marchetti GF, Ellis JL, Otis L, Sparto PJ, Watzlaf V, Whitney SL. Effect of home-based rehabilitation on activities of daily living and gait in older adults with heart failure at risk for falling: A retrospective cohort study. Physiother Theory Pract. 2017;21:1-11.

20. Yancy CW, Jessup M, Bozkurt B, Butler J, Casey DE, Jr., Drazner MH, Fonarow GC, et al. 2013 ACCF/AHA guideline for the management of heart failure: a report of the American College of Cardiology Foundation/American Heart Association Task Force on Practice Guidelines. J Am Coll Cardiol. 2013;62(16):e147-239.

21. Oudejans I, Mosterd A, Zuithoff NP, Hoes AW. Comorbidity drives mortality in newly diagnosed heart failure: a study among geriatric outpatients. J Card Fail. 2012;18(1):47-52.

22. Owan TE, Hodge DO, Herges RM, Jacobsen SJ, Roger VL, Redfield MM. Trends in prevalence and outcome of heart failure with preserved ejection fraction. $\mathrm{N}$ Engl J Med. 2006;355(3):251-259.

23. Kitada S, Kikuchi S, Tsujino T, Masuyama T, Ohte N, investigators JMs. The prognostic value of brain natriuretic peptide in patients with heart failure and left ventricular ejection fraction higher than $60 \%$ : a sub-analysis of the J-MELODIC study. ESC Heart Fail. 2017.

24. Forman DE, Sanderson BK, Josephson RA, Raikhelkar J, Bittner V, American College of Cardiology's Prevention of Cardiovascular Disease S. Heart failure as a newly approved diagnosis for cardiac rehabilitation: challenges and opportunities. J Am Coll Cardiol. 2015;65(24):26522659. 